\title{
Equidad y reformas de los sistemas de salud en Latinoamérica
}

\author{
Equity and health systems reform \\ in Latin America
}

Ingrid Vargas 1

Maria Luisa Vázquez 2

Elisabet Jané 2

\footnotetext{
1 Servicio de Estudios, Consorcio Hospitalario de Cataluña Av. Tibidabo 21,08022 Barcel ona, España. 2 CHC Consultoria i Gestió S. A. Av. Tibidabo 21, 08022 Barcel ona, España.
}

Abstract The aim of any heal th care system is to help improve the people's heal th, and to do so as efficiently as possible. In order to improve the efficiency and equity of health services provision, many countries around the world have implemented reforms, including several Latin American nations. However similar the objectives may appear, the vari ous ways soci eties implement such reforms reflect different values and concepts. This article analyzes the egalitarian and neoli beral values underlying different concepts of equity in health care. The authors devel op criteria to interpret selected health services funding and provi sion strategies in Latin American health system reforms. These criteria are then applied to health care financing and delivery policies under the reforms currently being implemented in Colombi a and Costa Rica.

Key words Equity; Heal th Care Reform; Health System; Health Financing

Resumen El fin último de cualquier sistema de salud es contribuir a la mejora dela salud de la población y hacerlo de la manera más eficiente posible. Buscando mejorar las condi ciones de eficiencia y equidad en que se prestan los servicios de salud, numerosos países en todo el mundo, incluyendo los latinoamericanos, han implementado reformas. A pesar dela aparente coincidencia en los obj etivos de las reformas, la forma en que se implementan responden a conceptos y valores diferentes. En este artícul o anal izamos los valores, igual itarios y neoliberales, subyacentes en los distintos conceptos de equidad. A partir de el los desarrollamos criteri os que nos permitan interpretar al gunas de las estrategias, financiamiento y prestación de los servi cios de salud aplicados por las reformas de los sistemas de sal ud en Latinoamérica. Estos criterios son aplicados a las políticas de financiamiento y prestaciones de las reformas apli cadas en los sistemas de salud de Colombia y Costa Rica.

Palabras clave Equidad; Reform en Atención dela Salud; Sistema de Salud; Financiamiento de la Salud 


\section{Introducción}

El fin último de cualquier sistema de salud es contribuir a la mejora de la salud de la población y a un aumento en la esperanza de vida. Aunque cada país organiza su sistema de salud de forma diferente, todos coinciden en la búsqueda continua de mejores formas de financiar, organizar y proveer los servicios de atención a la salud.

En las décadas de los ochenta y noventa, los gobiernos de muchos países, incluyendo los de Latinoamérica, iniciaron reformas de sus sistemas de salud. Estas reformas pretenden obtener mejoras en cuanto a la equidad, eficiencia y eficacia de los sistemas de salud (Berman \& Bir, 1995). Sin embargo, estos objetivos, aparentemente comunes, responden a conceptos cuya definición e interpretación dependen de los valores dominantes en la sociedad en que se traten. Por tanto, la interpretación de los mismos puede ser muy diferente en cada país.

La búsqueda de la equidad, en particular, es uno de los elementos centrales de los procesos de reforma del sector salud (Solimano et al., 1999). Una de las cuestiones fundamentales que se han debatido en torno al objetivo de equidad es la de si todos los individuos deben tener el mismo acceso a los servicios de salud de acuerdo con sus necesidades (enfoque igualitarista), o si se debería definir un conjunto limitado de servicios básicos de salud de acceso gratuito para los grupos de población con menos recursos (enfoque de niveles mínimos). A pesar de que la igualdad en el derecho a la salud, al acceso y uso de los servicios de salud figura como principio rector de la mayoría de las reformas de los países de América Latina, sólo algunos países de la región (Chile, Costa Rica y Cuba) han alcanzado tasas universales (o casi universales) de cobertura de salud (Homedes \& Ugalde, 1999).

En este artículo, hacemos un análisis de los enfoques subyacentes a los distintos conceptos de equidad. A partir del análisis de los conceptos, definimos criterios que nos ayuden a interpretar los objetivos de equidad en salud que se encuentran detrás de algunas de las estrategias de las reformas de los sistemas de salud en Latinoamérica. Para ello analizamos los modelos de financiación de los sistemas de saIud de Colombia y Costa Rica, aplicando los criterios previamente definidos. Finalizamos con algunas reflexiones sobre la manera en que podrían mejorarse los objetivos de equidad.
Concepto de equidad

y las teorías de justicia

El concepto de equidad se relaciona con aquello que consideramos “justo" y, por tanto, su significado depende de los valores o enfoques de sociedad, justicia social, que adopten los individuos, grupos o gobiernos (Barr, 1993). Para el análisis de la equidad en las políticas de salud, la diferencia más relevante se encuentra entre el enfoqueigualitarista, que formula los objetivos de equidad en términos de igualdad, y el enfoque neoliberal, que los expresa en términos de niveles mínimos y maximación del bienestar agregado.

El enfoque igualitarista se apoya en el concepto de justicia social de la filosofía comunitaria, que considera la solidaridad y la unidad social como algo bueno en sí mismo (Barr, 1993; Wagstaff \& van Doorslaer, 1992). Se prima la distribución de los recursos para alcanzar la igualdad de bienestar o la igualdad de recursos, por encima de los objetivos de maximización del bienestar económico de los individuos basados exclusivamente en criterios de eficiencia, que pueden conducir a la desigualdad entre grupos de población. Las políticas de salud cuyos objetivos de equidad están expresados en términos igualitaristas se dirigen a la eliminación de las desigualdades en los niveles de salud de la población. En este caso, se tenderá a sistemas de salud financiados públicamente y basados en el principio decapacidad depago. La aportación del ciudadano debe estar relacionada con cuánto puede pagar y no con su consumo de servicios de salud o con cualquier otra variable. Un ejemplo de este tipo son las políticas que persiguen la universalización de la cobertura del sistema de salud de un país, con independencia de la capacidad de pago.

El enfoque neoliberal de la justicia social parte de tres principios básicos: (1) el individualismo; (2) el igualitarismo, entendido como la igualdad de oportunidades y no la igualdad de resultados; y (3) la libertad, que se contempla como el derecho que tiene todo individuo a orientar su ámbito privado de acuerdo a sus propios intereses y preferencias. Los conceptos de justicia social que reflejan los valores neoliberales son los propuestos como niveles mínimos y la maximación del bienestar agregado (Pereira, 1988).

El enfoque de niveles mínimos, desarrollado extensamente por Rawls (1972), está firmemente anclado en la tradición liberal. Bajo este enfoque se justifica la acción pública en términos desiguales sólo para la mejora de las condiciones de la población más desfavorecida 
(Pereira, 1988). Por encima de los estándares mínimos, los individuos han de ser libres para procurarse en el mercado todos los bienes adicional es que deseen comprar. Por lo tanto, las políticas de salud basadas en estos valores se dirigen a garantizar a la población pobre un nivel básico mínimo de asistencia en salud.

El principio de justicia social que propugna el enfoque utilitarista es el de la maximización del estado de salud agregado dela población, sin importar su distribución. Esto implica que la acción pública se concentrará en los individuos con una mayor capacidad de beneficio, es decir, aquellos que puedan incrementar su nivel de salud (jóvenes). Uno de los instrumentos para llevar a la práctica el objetivo utilitarista de equidad es el análisis de coste-efectividad (Reich, 1995). Según este enfoque, las políticas con un mayor impacto sobre la salud de la población, medida en años de vida ajustados por discapacidad (AVADs) y en años de vida ajustados por calidad, (AVACs), serían aquellas que financian los tratamientos con un coste por AVADs más bajo (World Bank, 1993).

Este enfoque subyace en la política defendida por el Banco Mundial (World Bank, 1993), de financiar con fondos públicos un paquete mínimo de prestaciones, integrado por las intervenciones más costo efectivas, y dejar libertad al individuo para que se financie de forma privada el resto de prestaciones, entre las que se incluiría la atención curativa.

El enfoque neoliberal sobre la equidad apunta, por tanto, hacia una financiación de la salud esencialmente privada, apoyándose en el principio de capacidad de beneficio, de manera que cada ciudadano pague en función del beneficio que le reporta el consumo de servicios fruto de su elección individual. Este principio de capacidad de beneficio se concentra exclusivamente en objetivos de eficiencia económica, es decir en la maximización del beneficio individual, ignorando objetivos sociales como la redistribución de los recursos (Albi, 1992).

En resumen, los enfoques igualitaristas y neoliberales de justicia social darán lugar a sistemas de salud financiados de manera diferente y con consecuencias distintas en cuanto a la distribución final de los recursos y de los resultados de salud en los diversos grupos sociales.

Equidad en la financiación y en la prestación de servicios de salud

El análisis de la equidad en las políticas de reforma de los sistemas de salud lo realizaremos en base a dos aspectos fundamentales, por un lado, la equidad en la financiación, es decir, en la captación de los recursos y, por otro, la equidad en la prestación de servicios de salud, es decir, en la utilización de los recursos. Para ello, revisaremos primero el debate sobre los conceptos y criterios que vamos a aplicar posteriormente a dos casos concretos de reformas.

\section{La equidad en la financiación}

Desde el punto de vista de la equidad en la financiación, los igualitaristas distinguen entre equidad horizontal y equidad vertical (Wagstaff et al., 1992).

La equidad horizontal es el tratamiento igual para iguales. Es decir, que aquellos que poseen recursos iguales realicen pagos iguales, independientemente del sexo, estado civil, lugar de residencia, raza, etc. La inequidad horizontal se produce cuando individuos con igual renta contribuyen de forma diferente de acuerdo con ciertas características que no tienen que ver con su capacidad de pago. Podemos encontrar varias fuentes de inequidad horizontal en la financiación como, por ejemplo, la coexistencia de muchos seguros de enfermedad obligatorios con esquemas contributivos diferentes. En este modelo de financiación, dos individuos con la misma capacidad de pago pueden estar realizando aportaciones diferentes, dependiendo del fondo al que estén afiliados.

La equidad vertical se define como el tratamiento desigual para desiguales. Aplicado a la financiación significa que aquellos con desigual capacidad de pago deben realizar pagos distintos al sistema, de forma que aquellos que más recursos tienen deben contribuir al sistema más que proporcionalmente a su nivel de renta. La equidad vertical de la financiación implica la redistribución final neta de la renta entre los miembros de una sociedad, ya que saldrán recursos de los más ricos hacia los más pobres. La equidad vertical de una fuente de financiación equivale a la progresividad de la misma. Una fuente de financiación progresiva es aquélla en la que las contribuciones que realiza un individuo como proporción de su renta aumentan conforme aumenta su renta. La equidad vertical de la financiación de un sistema de salud se mi de con índices de progresi vidad como el índice Kakwani a partir de encuestas de hogares (Kakwani, 1977). Este índice compara la renta que percibe cada decila de población con la contribución a la financiación de la salud que realiza. Si el sistema de financiación es progresivo, los más pobres pagan relativamente menos en comparación con su renta y los más ricos relativamente más. 
Según un estudio sobre la equidad en la financiación, realizado en diez países europeos utilizando el índice de Kakwani, en orden de progresividad, encontraríamos las siguientes fuentes: impuestos, seguridad social, seguro privado y pagos directos (Wagstaff et al., 1992). Este orden se cumple en la mayoría de los países analizados. Sin embargo, todas las fuentes de financiación pueden presentar elementos que disminuyen su progresividad o regresividad, según sea el caso (Tabla 1).

Debido a que el sistema de financiación de salud de un país está compuesto por más de una fuente (impuestos, seguros obligatorios, etc.), la equidad vertical de todo el sistema dependerá de la progresividad de cada una de las fuentes y de la proporción en que cada fuente contribuya a la financiación de la sanidad.
La equidad en la prestación

de servicios de salud

Desde la óptica igualitarista, tres son fundamentalmente las definiciones de equidad en la prestación de la asistencia sanitaria: igual tratamiento para igual necesidad, igualdad de acceso e igualdad de salud (Wagstaff et al., 1991).

El criterio de igualdad de tratami ento para igual necesidad significa que las personas con igual necesidad de atención sanitaria reciben el mismo tratamiento independientemente de cualquier característica personal que no tenga que ver con la necesidad (capacidad de pago, sexo, lugar de residencia, etc). El problema en la aplicación de este criterio reside en la dificultad de la definición de necesidad y tratamiento.

Tabla 1

Factores que inciden en el nivel de progresividad de las fuentes de financiación de los sistemas de salud.

\begin{tabular}{|c|c|c|c|}
\hline Fuente de financiación & Nivel de progresividad & $\begin{array}{l}\text { Factores que mejoran la } \\
\text { progresividad de la fuente }\end{array}$ & $\begin{array}{l}\text { Factores que disminuyen la } \\
\text { progresividad de la fuente }\end{array}$ \\
\hline $\begin{array}{l}\text { Impuesto directo sobre } \\
\text { la renta }\end{array}$ & $\begin{array}{l}\text { Fuente de financiación más } \\
\text { progresiva (individuos con más } \\
\text { renta pagan más que } \\
\text { proporcionalmente) }\end{array}$ & $\begin{array}{l}\text { - Tipo impositivo creciente } \\
\text { con la renta } \\
\text { - Mínimos exentos de impuesto } \\
\text { para niveles de renta de } \\
\text { subsistencia }\end{array}$ & $\begin{array}{l}\text { - Fraude fiscal } \\
\text { - Tamaño elevado del trabajo informal } \\
\text { (evasión) } \\
\text { - Procesos inflacionistas } \\
\text { - Desgravaciones en la base imponible }\end{array}$ \\
\hline $\begin{array}{l}\text { Contribuciones obligatorias } \\
\text { a seguros sociales }\end{array}$ & $\begin{array}{l}\text { Fuente de financiación } \\
\text { proporcional (porcentaje } \\
\text { del salario) }\end{array}$ & $\begin{array}{l}\text { Exención de la cotización de la } \\
\text { población con niveles de renta } \\
\text { de subsistencia }\end{array}$ & $\begin{array}{l}\text { - Evasión y subcotización (tamaño de la } \\
\text { economía informal, desempleo) } \\
\text { - Topes máximos en los tramos } \\
\text { de cotización } \\
\text { - Traslación de la cuota del empresario } \\
\text { al salario del trabajador o al precio de } \\
\text { bienes de consumo }\end{array}$ \\
\hline Impuestos indirectos & $\begin{array}{l}\text { Fuente de financiación regresiva } \\
\text { (peso mayor del consumo en las } \\
\text { rentas bajas) }\end{array}$ & $\begin{array}{l}\text { - Tipo impositivo reducido sobre } \\
\text { artículos de primera necesidad } \\
\text { (baja elasticidad-ingreso) } \\
\text {-Tipo impositivo elevado sobre } \\
\text { bienes de lujo (elevada elasticidad- } \\
\text { ingreso) }\end{array}$ & $\begin{array}{l}\text { - No se aplican tipos impositivos } \\
\text { diferentes según el tipo de bien }\end{array}$ \\
\hline Seguros de salud privados & $\begin{array}{l}\text { Fuente de financiación regresiva } \\
\text { (precio independiente de la } \\
\text { capacidad de pago; mayor para } \\
\text { los riesgos elevados que suelen } \\
\text { coincidir con los individuos con } \\
\text { menor renta. }\end{array}$ & $\begin{array}{l}\text { - Seguros privados suplementarios } \\
\text { cuya cartera de servicios coincide } \\
\text { con la pública (bien de lujo) }\end{array}$ & $\begin{array}{l}\text { - Seguros privados complementarios al } \\
\text { público (cartera de los servicios que } \\
\text { no cubre el seguro público) }\end{array}$ \\
\hline $\begin{array}{l}\text { Pagos directos } \\
\text { (out of pocket) }\end{array}$ & $\begin{array}{l}\text { Fuente de financiación regresiva } \\
\text { (gasto privado como porcentaje } \\
\text { del gasto total mayor para hogares } \\
\text { de renta baja) }\end{array}$ & $\begin{array}{l}\text { - Estructura de precios con } \\
\text { distintos tramos } \\
\text { - Exenciones en función de la renta }\end{array}$ & \\
\hline
\end{tabular}

Fuente: Elaboración propia. 
La igualdad de acceso debería interpretarse como la igualación de los costes en que han de incurrir los pacientes para obtener atención médica de una determinada calidad (Le Grand, 1982). Según esta definición, se considerarían como costes de acceso: los precios de los servicios, los costes de transporte y el coste del tiempo.

El criterio de igualdad desalud resulta aún más problemático, debido a la influencia que en la salud tienen otros factores, como la educación y la renta, sobre los que desde los servicios de salud no se tiene influencia. A esto se añade la dificultad de medir los niveles de salud.

Las tres definiciones igualitaristas de equidad en la prestación no son equivalentes. Por un lado, el acceso al tratamiento es un fenómeno de la oferta, mientras que el tratamiento que finalmente se recibe es un fenómeno de la interacción entre oferta y demanda, es decir, el tratamiento no sólo depende de los costes de acceso sino también de la percepción del paciente sobre los beneficios de la asistencia sanitaria. Dos individuos pueden tener el mismo acceso a los servicios médicos y, sin embargo, uno puede preferir ir al médico y recibir tratamiento y el otro no. Por otro lado, la salud del individuo, tal y como se ha comentado, no sólo depende del acceso a los servicios en salud o al tratamiento adecuado, sino que también inciden otros factores.

La aplicación de cualquiera de estas definiciones resulta difícil en la práctica. La mayoría de los autores en las obras consultadas prefieren las dos primeras definiciones de equidad en la prestación (Wagstaff et al., 1992), aunque concluyen que no existe un único modo correcto de definir la equidad.

Numerosos estudios empíricos (Hurst, 1985; Le Grand, 1978), han basado la medición de la equidad en la prestación de servicios en el concepto de igualdad de tratamiento para igual necesidad, independientemente de características personales, como la renta, que no guardan relación con la necesidad. El tratamiento se mide por el gasto y la necesidad por al guna variable de morbilidad. El grado de equidad se determina comparando las distribuciones de enfermedad y gasto por decila de renta, a través de la curva de concentración de la enfermedad y de la curva de concentración del gasto, respectivamente. Si el gasto en salud es equitativo, las dos curvas coincidirán, de manera que los individuos con las mismas necesidades de salud recibirán los mismos recursos, independientemente de su nivel de renta. Estos serán los conceptos y criterios de análisis que nosotros utilizaremos para analizar la equidad en la prestación de los países analizados.

\section{El concepto de eficiencia}

En términos generales, la noción de eficiencia se refiere al mejor uso de recursos escasos. Es necesario distinguir entre eficiencia técnica, económica y asignativa. La opción técnicamente eficiente es aquella que utiliza la menor cantidad de recursos para producir una determinada cantidad de bienes y servicios. La eficiencia económica se logra cuando, entre aquellas opciones técnicamente eficientes, se elige la que genera un menor coste. Finalmente, la eficiencia asignativa se alcanza cuando la elección, además de ser técnica y económicamente eficiente, es la que la sociedad más valora (Barr, 1993).

Para evaluar la eficiencia de la financiación de un sistema de salud es necesario tener en cuenta dos aspectos: en primer lugar, los costes asociados a la administración del sistema (eficiencia técnica) y, en segundo lugar, el grado de eficiencia económica del sistema de financiación. El segundo tipo de costes viene determinado por la distorsión que en el mercado puede producir la introducción de un impuesto o una contribución obligatoria, por ejemplo, en forma de mayores costes para las empresas, o por los fallos que llevan a que el mercado de seguros no sea eficiente, principalmente la selección adversa y el riesgo moral.

El tipo de costes que se tendrán en cuenta para medir el grado de eficiencia de la financiación del sistema de salud en los países analizados son los generados por la administración del sistema.

La equidad y las reformas del sector salud en Latinoamérica

Para analizar los objetivos de equidad de las políticas de reforma de los sistemas de salud, hemos elegido dos países latinoamericanos, CoIombia y Costa Rica, que han implementado modelos diferentes de sistemas de salud, pero con objetivos semejantes: Ia universalización de la cobertura. Ambos países, con un Producto Interno Bruto (PIB) de US\$2.600 y US\$2.780 per cápita, respectivamente, pertenecen al grupo de países con renta media baja (World Bank, 2000).

Para analizar la equidad en las políticas de reforma del sistema de salud de Colombia y Costa Rica, valoraremos las fuentes de financiación y el peso que tiene cada fuente en la estructura de financiación del sistema de salud, por un lado, y, por otro, la cobertura de las prestaciones, así como el gasto público en salud realizado según decilas de renta. 


\section{Colombia}

En Colombia, la Constitución de 1991 reconoce explícitamente la salud como un derecho inalienable de los ciudadanos y define la seguridad social como un servicio público que debe ser provisto bajo la dirección del Estado, en base a los principios de universalidad, eficiencia y solidaridad. En 1993, fue promulgada la Ley 100 (Ministerio de Salud, 1993), por la cual se crea el Sistema General de Seguridad Social en Salud (SGSSS). Esta ley establece los principios generales del nuevo sistema en cuanto a regulación, financiación, organización y prestación de servicios de salud.

En cuanto a la financiación de los servicios de salud, se creó un Fondo Nacional de Salud (Fondo de Solidaridad y Garantia), que debía absorber todos los fondos anteriores. Este, a su vez, está divido en cuatro subcuentas, dos destinadas a la atención a salud: subcuenta del régimen contributivo y subcuenta del régimen subsidiado (SUPERSALUD, 1996). La población, por su parte, estará afiliada a un régimen de carácter contributivo o subsidiado, que da derecho a diferentes tipos de prestaciones. El régimen contributivo para asalariados y trabajadores independientes, da derecho a un Plan Obligatorio de Salud (POS) y a la posibilidad de ampliar sus beneficios con la compra de un plan complementario. El POS integra servicios básicos para el cuidado: de prevención, promoción de salud, atención de primer nivel y riesgos catastróficos. El régimen de seguro subsidiado da acceso a un plan (POS-S) que, inicialmente, sólo cubre los cuidados primarios de salud, orientados a combatir las enfermedades transmisibles y proveer la atención materno infantil (Londoño, 1996). No obstante se han incluido algunos procedimientos terapeuticos y reaseguro para enfermedades de alto costo (Jaramillo-Perez, 1999), la intención es que la cartera de servicios de este plan converja a la que cubre el plan obligatorio del régimen contributivo. El régimen subsi diado está destinado a la población más pobre, que, según datos de la Comisión Económica para América Latina y el Caribe (CEPAL), ascendió en 1997 al 45\% de los hogares colombianos (Ocampo \& Franco, 2000).

La gestión del aseguramiento, incluyendo la afiliación y la recaudación de las cotizaciones, ha sido descentralizada a las Empresas Promotoras de Salud (EPS), que pueden ser de diversa índole: estatal, sin ánimo de lucro o privadas, responsables también de la compra de los servicios de salud a los proveedores (Londoño, 1996).
La equidad en la financiación del sistema colombiano de salud

Existen diversos elementos en el modelo de financiación colombiano del sistema de salud que influyen en la equidad vertical en la financiación:

- Proporción del gasto privado en el gasto total en salud

El Estado colombiano en los últimos años, ha realizado un esfuerzo importante incrementando su aportación al sistema de salud (un 33\% entre 1994 y 1997; Restrepo, 1997). No obstante, el gasto privado efectuado por los hogares en Colombia sigue representando la mayor parte del gasto en salud, entre el $45,5 \%$ y el $58 \%$ del gasto nacional de salud, en 1997, según fuentes consultadas (Málaga et al., 2000; WHO, 2000). En los diversos estudios analizados, la financiación privada, los pagos directos y los seguros privados son la fuente de financiación más regresiva, debido a la relación inversa entre la posición socioeconómica y la salud del individuo: cuanto más baja es la renta del individuo, más servicios sanitarios demandará y, por lo tanto, más pagos realizará como proporción de su renta, ya sea en forma de póliza de seguros o mayores copagos (Wagstaff et al., 1992).

- Financiación del gasto público en salud

El $62,5 \%$ del gasto público en salud fue financiado con impuestos y el 37,5\% restante, con cuotas obligatorias de trabajadores y empresarios (WHO, 2000). Del total de impuestos, los indirectos representaron, en 1995, el 50,3\% de los ingresos corrientes, mientras que los directos fueron tan sólo el 35,5\%. Colombia posee un IVA redistributivo: por cada $1 \%$ del PIB en ingresos adicionales, se reduce la desigualdad en el ingreso, medida con el índice de Gini, en más del 0,4 puntos porcentuales, debido a la exención de los principales artículos de la canasta de consumo básico. No obstante, según un análisis hecho por el BID para Latinoamérica, se concluye que el potencial redistributivo de los impuestos indirectos siempre es inferior al del impuesto sobre la renta (BID, 1998).

\section{- Subcotización}

Esta viene determinada, en parte, por el importante peso del sector informal de la economía que, en 1998, afectaba a más del 55\% del empleo urbano (Ocampo \& Franco, 2000), y por la 
dificultad de determinar los ingresos reales de los trabajadores indepiendentes.

\section{- Financiación del régimen subsidiado}

En el sistema contributivo del SGSSS, el empleador cotiza el $8 \%$ y el asegurado el $4 \%$ de su salario, de esto $1 \%$ es para financiar el régimen subsidiado, lo cual añade progresividad al sistema. El régimen subsidiado está financiado, además de con este $1 \%$, con fondos procedentes de diversas fuentes a nivel nacional y territorial (impuestos indirectos, subsidio familiar, y otros ingresos de origen fiscal; SUPERSALUD, 1996). Cualquier déficit es cubierto por el Estado. Los pobres están exentos de todo pago al seguro subsidiado. Para su identificación, se han utilizado encuestas nacionales.

La equidad en la prestación del sistema colombiano

- La igualdad en el acceso a los servicios de salud

La reforma del sistema de salud en Colombia propone inicialmente un modelo de paquetes de prestaciones que segmenta el acceso a los servicios de salud en función de variables que no dependen de la necesidad de salud de los individuos, como son la capacidad de pago y el status laboral. Según estas variables, los hogares tienen derecho a paquetes de prestaciones más o menos amplios.

La meta inicial era que, en el 2001, toda la población estuviera cubierta por el POS (Londoño, 1996). En los primeros años de la reforma, la afiliación del aseguramiento público se ha incrementado hasta al canzar aproximadamente al $57 \%$ de la población, debido principalmente a la incorporación al seguro obligatorio de los familiares de los trabajadores afiliados. El 37,6\% de la población está afiliada al régimen contributivo, un $19,5 \%$, al régimen subsidiado y la población restante no está vinculada al sistema (Jaramillo-Perez, 1999). Teniendo en cuenta que, antes de la reforma, aproximadamente el $60 \%$ de la población (Londoño, 1996) era atendida por el Ministerio de Salud, es difícil valorar el aumento real de acceso a los servicios por parte de la población. Estas cifras nos hablan de la cobertura en cuanto a afiliación, pero no nos dicen nada de la cobertura en cuanto a acceso a los servicios de salud (Mesa-Lago, 2000). Adicionalmente, existe la sospecha de que un número importante de afiliados (13\%) lo estén en varias EPS simultáneamente (Málaga et al., 2000).
Para aumentar la cobertura del seguro social, se han introducido incentivos a las EPS mediante un pago capitativo que vincula sus ingresos con el número de personas apuntadas a su lista. Para evitar que las aseguradoras tengan incentivos a seleccionar riesgos, descartando la afiliación de aquellos trabajadores pobres y con más probabilidad de utilizar los servicios, el pago capitativo se ha ajustado por variables como el sexo y la edad, y se ha impedido que las organizaciones puedan rechazar las solicitudes de afiliación (Medici et al., 1997). A pesar de estos controles, la aseguradoras podrian emplear múltiples mecanismos para rechazar los altos riesgos excluyéndolos del aseguramiento obligatorio: ofrecer a este tipo de individuos servicios de salud de baja calidad, no atender sus reclamaciones, contratar proveedores que sólo actúen en "zonas geográficas saludables", u ofrecerles sumas de dinero para que contraten un seguro con otra aseguradora.

Por otro lado, la evidencia en el uso de fórmulas de ajuste de riesgo en Estados Unidos y en Holanda muestra que incluso la metodología más sofisticada explica menos de la mitad de la varianza del coste futuro de la atención de salud. Con un grado de predicción tan bajo, el margen para que se den incentivos a seleccionar sólo los individuos con menos probabilidad de enfermedad es demasiado alto.

La extensión del aseguramiento obligatorio al resto de la población parece difícil, si tenemos en cuenta que el tipo de mecanismo empleado por la reforma para conseguir una mayor afiliación presenta problemas lo suficientemente importantes como para incrementar aún más la desigualdad de acceso de partida del sistema de salud.

- La igualdad de gasto para igual necesidad de salud

Si analizamos la distribución del gasto público en salud en Colombia, podemos observar que los quintiles de renta que más se benefician del gasto público en salud son el III y el IV, percibiendo el $22,2 \%$ y el $20,7 \%$ del gasto, respectivamente. El $20 \%$ de hogares más pobres recibe un $17,5 \%$ del gasto en salud y nutrición, mientras que la proporción destinada al $20 \%$ de hogares más ricos es mayor, un 19,7\% (Ocampo \& Franco, 2000). Puesto que la enfermedad se concentra en los grupos con ingresos más bajos, se produce una inequidad en el gasto que favorece a los ricos (Figura 1 ). 
Figura 1

Distribución del gasto social en salud por quintiles de ingreso familiar.

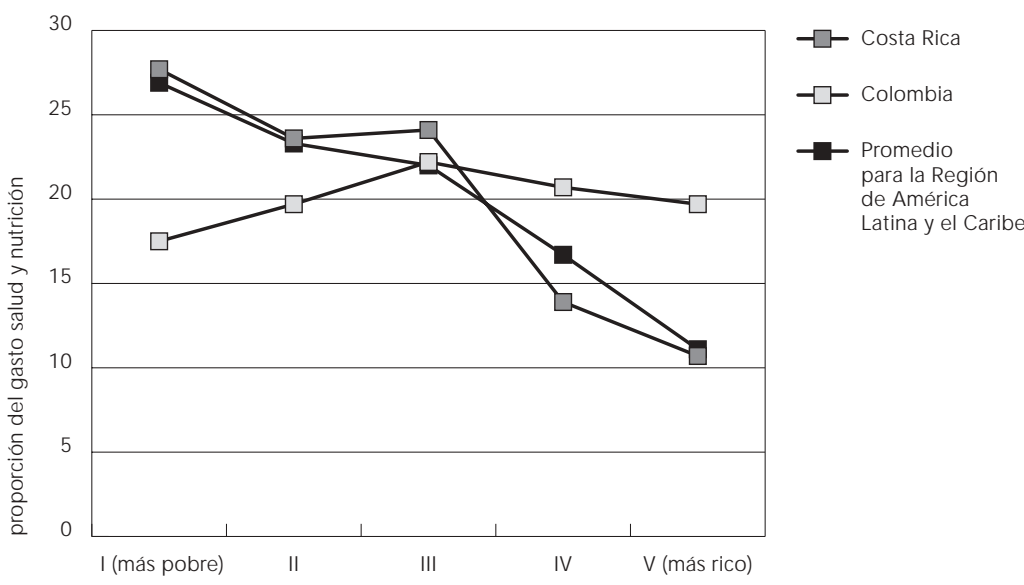

La eficiencia en la financiación del sistema colombiano de salud

El número de EPS que competían por el aseguramiento era, aproximadamente, de 30 para un total de 14 millones de afiliados (Medici et al., 1997). En un modelo de salud de múltiples pagadores, se elevan los costes de transacción y de administración del sistema. Los costes de transacción vienen determinados por la cantidad de contratos que se establecen entre las administradoras y los proveedores. Los costes de administración pueden ser de dos tipos: aquellos costes asociados a la afiliación, producción y comercialización de seguros, que recaen sobre la empresa aseguradora, y los costes derivados de la administración de múltiples seguros privados, que recaen en los proveedores de servicios sanitarios. Por ejemplo, la existencia de múltiples pagadores impone altos costes burocráticos a los proveedores, que han de gestionar numerosos contratos, planes que cubren diferentes servicios, copagos, documentación, etc. Woolhandler \& Himmelstein (1991) han estimado que los costes administrativos de un modelo sanitario de múltiple pagador como el americano ascienden a un 19 - 24\% de su gasto en salud, mientras que, en un modelo de único pagador como el canadiense, los costes administrativos equivalen a un 8 - $11 \%$ del gasto. En Colombia, el gasto en salud aumentó de $6 \%$ del PIB, en 1993, a alrededor del $10 \%$ del PIB, en 1999 (Málaga et al., 2000). Este aumento del gasto en salud, sin que haya aumentado el acceso a los servicios de salud, parece indicar que en el sistema existen ineficiencias.

\section{Costa Rica}

En los años setenta, Costa Rica emprendió reformas en su sistema de salud que culminaron en la década de los noventa. La principal peculiaridad de la reforma costarricense ha sido la transferencia de los servicios de atención a la salud de las personas desde el Ministerio de Salud a la Caja Costarricense de Seguro Social (CCSS), proceso que se inició en la década de los setenta con la transferencia de los hospitales y que ha culminado en la actualidad con la atención primaria de salud.

En el Plan Nacional de Desarrollo se establece que el Estado asegurará las condiciones favorables para mejorar la salud y proveer los servicios. Asimismo, se establecen los principios sobre los que se debe desarrollar el sistema de salud: solidaridad en el financiamiento, equidad en la cobertura, calidad y eficiencia (OPS, 1998). El Ministerio de Salud detenta las funciones rectoras y de promoción de la salud, mientras que la CCSS se ocupa de la atención a la salud de las personas, es decir, de las actividades de promoción, prevención, recuperación y rehabilitación de la salud. La CCSS es un seguro de salud único, con cobertura universal (Güendel \&Trejos, 1994).

La financiación del seguro de enfermedad y maternidad se hace mediante cotizaciones sobre el salario, siendo la afiliación al seguro voluntaria para los trabajadores independientes. Todos los costarricenses, por el hecho de serlo, tienen acceso a las mismas prestaciones de servicios de salud por parte de la CCSS.

En cuanto a la organización de los servicios se han priorizado la extensión y el fortalecimiento de la atención primaria de salud, enfatizando las intervenciones de prevención y promoción y dotando equipos básicos de atención integral a la salud de mayor capacidad de resolución en el campo curativo (Jané, 1999).

La equidad en la financiación del sistema costarricense de salud

En Costa Rica, como en Colombia, diversos elementos influyen en la equidad de la financiación del sistema de salud:

- Proporción del gasto privado en el gasto total en salud

La financiación pública representa el $77,1 \%$ del gasto total en salud (WHO, 2000). La fuente de fi- 
nanciación privada mayoritaria es el pago directo que supone el $22,3 \%$ del gasto total en salud.

\section{- Financiación del gasto público en salud}

La principal fuente de financiación son las contribuciones al seguro de enfermedad, que suponen el $83,8 \%$. El 16,2\% restante se financia con impuestos, el 77,1\% de los cuales son indirectos (BID, 1998). Los aportes del Estado a la CCSS son irregulares.

\section{- Subcotizaciones}

En la década de los 90, se produjo un aumento del peso del empleo informal, que en 1997 representaba casi el $40 \%$ del empleo total, con lo cual se ha incrementado la subcotización y evasión al seguro (Ocampo \& Franco, 2000). Este factor, junto a la disminución de la afiliación a la seguridad social entre los ocupados, tiene un efecto negativo sobre la equidad de la financiación del sistema de salud.

- Contribuciones al sistema de seguridad social

La financiación del seguro de enfermedad y maternidad se hace mediante cotizaciones del $14 \%$ sobre el salario. El pago de las cotizaciones depende de si los trabajadores son asalariados o independientes (asegurados voluntarios). En el caso de los asalariados, la cotización es tripartita: 9,25\% del aporte sobre el salario corre a cargo del empresario, el trabajador contribuye con el 5,5\% y el Estado con un 0,25\%. Los asegurados voluntarios aportan 13,25\%, mientras que el Estado aporta el 0,25\%.

La equidad en la prestación del sistema costarricense de salud

- Igualdad en el acceso a los servicios de salud

Las prestaciones que ofrece la CCSS son iguales para todos los afiliados con independencia del valor de la contribución. No obstante, existe diferencia en la oferta de servicios según áreas geográficas y, por tanto, no todos los servicios están accesibles de igual manera en todas las áreas del país. Así, por ejemplo, la estrategia de fortalecer el primer nivel de atención tiene la meta de construir 800 sectores de salud con equipos básicos de atención integral a la salud (EBAIS). Este modelo ya está implantado en las zonas rurales, pero aún está adaptandose para las zonas urbanas.
Otra fuente de inequidad en el sistema es la relacionada con los inmigrantes. En Costa Rica hay entre 500.000 y 800.000 inmigrantes, no todos con sus papeles en regla, cuyo acceso a los servicios de salud es muy variable. Se les garantiza el acceso a lo que se podría denominar paquete básico, pero las otras prestaciones que pueden recibir varían según su status legal, lugar de residencia y trabajo (Jané, 1999).

\section{- Igualdad de gasto para igual} necesidad en salud

En cuanto a la redistribución del gasto en salud, el $20 \%$ de los hogares con menores ingresos es el que más se beneficia del gasto público en salud y nutrición, ya que percibe el $27,7 \%$ del gasto total en salud. La proporción del gasto que reciben los hogares más ricos, último quintil de renta, es sustancialmente inferior: un $10,7 \%$ del gasto total (Ocampo \& Franco, 2000; Figura 1).

La eficiencia en la financiación del sistema costarricense de salud

Dos de los factores que explican la existencia de costos más bajos en los regímenes públicos son los bajos costos de mercadeo y afiliación y la presencia de fuertes economías de escala en la mayor parte de los procesos que constituyen la administración del sistema de seguro social (Rodríguez \& Durán, 2000). Las economías son menores en los regímenes privados en cuya administración participa un número mayor de gestores. Un gasto total en salud de un $8,7 \%$ del PIB (WHO, 2000), con una cobertura casi universal de los servicios de salud, podría ser un indicio de un sistema eficiente.

\section{Discusión}

El principio de equidad que se expresa en la Constitución colombiana es el de igualdad en el acceso a los servicios de salud que deben ser responsabilidad pública (aunque no necesariamente de su propiedad). El derecho colectivo es a la salud y no sólo a la protección de la misma. A pesar de que este principio de equidad expresado en la Constitución es igualitarista, en la práctica, se ha establecido un Sistema de Seguridad Social basado en un enfoque liberal de la equidad. En primer lugar, se ha segmentado el acceso a los servicios de salud en función de la capacidad de pago del individuo y de su status laboral. En segundo lugar, en el diseño del POS y del plan subsidiado, se han em- 
pleado instrumentos de costo-efectividad, siguiendo un enfoque utilitarista de equidad.

En cuanto a la financiación, se pueden identificar adicionalmente diversas fuentes de inequidad. La principal causa de la regresividad del modelo de financiación colombiano viene determinada por el importante peso de la financiación privada de la asistencia sanitaria (más de la mitad de la misma), ya que el gasto privado en salud es proporcionalmente mayor en los grupos de renta baja, que además son los que más enferman (Fernández et al., 2000). Otro factor que contribuye a incrementar la inequidad en la financiación del sistema de salud es el aumento de la subcotización, debido a la extensión del empleo informal.

En cuanto a las prestaciones, la reforma del sistema de salud en Colombia propone inicialmente un modelo que es inequitativo, ya que segmenta el acceso a los servicios de salud en función de variables que no dependen de la necesidad de salud de los individuos, como son la capacidad de pago y el status laboral. La igualdad en el acceso a los servicios de salud en Colombia dependerá, en cierta medida, de que se logren los objetivos de extender el aseguramiento obligatorio. No obstante, el mecanismo utilizado con este propósito, basado en la desconcentración del aseguramiento a las EPS, crea incentivos contrarios.

A pesar de que, en el último informe de la Organización Mundial de la Salud (WHO, 2000), Colombia aparece como el país más equitativo en la financiación de su sistema de salud, el presente análisis indica que existen elementos suficientes en contra de esta afirmación. Probablemente sea debido a que el indicador utilizado (razón entre contribuciones a la financiación en salud y el gasto total en bienes no alimentarios) no puede proporcionar información sobre la progresividad de los pagos de los individuos, a menos que se conozca cómo crece el gasto en bienes no alimentarios con la renta. Por otro lado, tal y como se ha explicado en las secciones anteriores, la equidad en la financiación viene determinada no sólo por cómo se captan los recursos (equidad en la financiación), sino también por cómo se los utilizan (equidad en el gasto), y nuestro análisis parece indicar inequidades en este respecto.

En Costa Rica, se parte de principios igualitarios de la equidad y se logra una buena aproximación a los mismos. Por un lado, existe una financiación equitativa del sistema de salud debido, fundamentalmente, al importante peso de la financiación pública en la misma. Junto con la educación primaria, el gasto en salud es el rubro más redistributivo y de mayor efec- to desconcentrador en la distribución del ingreso. Esto reafirma la tesis de que el gasto público social es el principal instrumento a través del cual el Estado influye sobre la distribución del ingreso (Ocampo \& Franco, 2000).

Por otro lado, una de las políticas que más han contribuido a la equidad de acceso a los servicios de salud en Costa Rica ha sido la extensión y fortalecimiento de la atención primaria de salud (Jané, 1999). Esta estrategia ha ayudado a revertir la desigualdades de acceso generadas por la alta concentración de recursos en algunas regiones del país (especialmente en el Valle Central) y en la atención hospitalaria.

No obstante, también hay elementos de inequidad en el sistema. La principal fuente de desigualdades en el sistema de salud costarricense se encuentra en el acceso a la prestación de los servicios de salud. Son debidas a la distribución geográfica de los servicios, con mayor concentración en el área urbana, la desigual distribución de la atención primaria y especializada, así como la cobertura desigual a la población de inmigrantes.

\section{Conclusiones}

Los principios de equidad expresados por ambos países se basan en enfoques igualitarios, y definen objetivos de universalización de los sistemas de salud. No obstante, los mecanismos para universalizar el aseguramiento público utilizados en ambos países han sido distintos y con resultados diferentes. En el caso de Colombia, se ha buscado extender el aseguramiento, con una oferta de servicios desigual, según la capacidad adquisitiva de la población. Debido a la magnitud de la pobreza, de la economía informal y de los incentivos a la selección de riesgos, es improbable que, a medio plazo, se alcance el objetivo de la universalización del aseguramiento obligatorio con igualdad de prestaciones.

En Costa Rica, por el contrario, se ha optado por la financiación pública del sistema de salud, con igualdad de prestaciones, con independencia de la capacidad adquisitiva. Esto ha permitido la puesta en práctica de los principios igualitarios de la equidad, con una redistribución del gasto hacia los sectores más necesitados. A pesar de que aún mantiene algunas características de desi gualdad, como diferente acceso según lugar de residencia y procedencia, se puede decir que ha logrado un sistema equitativo igualitario. 


\section{Referencias}

ALBI, E.; CONTRERAS, C.; GONZALEZ-PARAMO, J. M. \& ZUBIRI, I., 1992. Teoría de la Hacienda Pública. 1a Ed. Barcelona: Editorial Ariel.

BARR, N., 1993. The Economics of theWelfare State. 2nd Ed. Oxford: Oxford University Press.

BERM AN, P. A. \& BIR, A., 1995. Introduction. In: Health Sector Reform in Developing Countries. Making Health Development Sustainable (P. A. Berman, ed.), pp. 1-13, Boston: Harvard University Press.

BID (Banco Interamericano de Desarrollo), 1998. Amé rica Latina Frentea la Desigualdad. Washington, DC: BID.

FERNANDEZ, E.; SCHIAFFINO, A.; RAJMIL, L.; BORRELL, C. \& SEGURA, A., 2000. Desigualdades en salud según la clase social en Cataluña, 1994. Atención Primaria, 25:96-102.

GÜENDEL, L. \& TREJOS, J. D., 1994. Reformas Recientes en el Sector Salud de Costa Rica. Serie Reformas de Política Pública 18. Santiago de Chile: Comisión Económica para América Latina y el Caribe, Naciones Unidas.

HOMEDES, N. \& UGALDE, A., 1999. Condiciones y condicionantes de salud y reforma. In: Foro Internacional: La Reforma del Sector Salud, Anales, pp. 137-147. Ciudad de Guatemala: Proyecto de Apoyo a la Reforma del Sector Salud.

HURST, J. W., 1985. Financing Health Services in the United States, Canada and Britain. London: King Edward's Hospital Fund.

JANÉ, E., 1999. Sistemas de salud y desarrollo. Quadern CAPS, 28:7-16.

JARAMILLO-PEREZ, I., 1999. El Futuro dela Salud en Colombia. Ley 100 de 1993 Cinco años Después. Bogotá: Frescol - Fes - FRB - Fundación Corona.

KAKWANI, N. C., 1977. Measurement of tax progressivity: An international comparison. Economic Journal, 87:71-80.

LE GRAND, J., 1978. The distribution of public expenditure: The case of health care. Económica, 45:125-142.

LE GRAND, J., 1982. Health care. In: The Strategy of Equality (J. Le Grand, ed.), pp. 23-53, London: Allen \& Unwin.

LONDOÑO, J. L., 1996. Managed Competition in the Tropics? Ottawa: World Bank. (mimeo.)

MALAGA, H.; LATORRE, M. C.; CARDENAS, J.; MONTIEL, H.; SAM PSON, C.; TABORDA, M. C.; GRANDADOS, R. \& PASTOR, D., 2000. Equidad y reformas en salud en Colombia. Revista de Salud Pública, 2:193-219.

MEDICI, A. C.; LONDOÑO, J. L.; COELHO, O. \& SAXENIAN, H., 1997. Managed care and managed competition in Latin America and the Caribbean. In: Innovations in Health CareFinancing (G. J. Schieb, ed.), pp. 215-231, Washigton, DC: The World Bank.

MESA-LAGO, C., 2000. Desarrollo Social, Reforma del Estado y dela Seguridad Social, al Umbral del Siglo XXI. Serie Políticas Sociales 36. Santiago de Chile: División de Desarrollo Social, Comisión Económica para América Latina y el Caribe, Naciones Unidas.

MINISTERIO DE SALUD, 1993. Ley 100 de 1993. La Seguridad Social en Colombia. Bogotá: Ministerio de Salud.

OCAMPO, J. A. \& FRANCO, R., 2000. La Brecha dela Equidad: Una Segunda Evaluación. Santiago de Chile: Comisión Económica para América Latina y el Caribe, Naciones Unidas.

OPS (Organización Panamericana de la Salud), 1998. Costa Rica. In: La Salud en las Américas (OPS, ed.), Publicación Científica 569, v. 2, pp. 198-211, Washington, DC: Organización Mundial de la Salud.

PEREIRA, J., 1988. La interpretación económica de equidad en salud y atención sanitarias. En salud y equidad, In: VIII Jornadas de Economía de la Salud, Anales, pp. 31-52. Madrid: Ministerio de Sanidad y Consumo.

RAWLS, J., 1972. A Theory of Justice. Oxford: Oxford University Press.

REICH, M. R., 1995. The politics of health sector reform in developing countries: Three cases of pharmaceutical policy. In: Health Sector Reform in Developing Countries. Making Health Development Sustainable (P. A. Berman, ed.), pp. 59-99, Boston: Harvard University Press.

RESTREPO, M., 1997. La Reforma a la Seguridad Social en Salud de Colombia y la Teoría dela Competencia Regulada. Serie Financiamiento del Desarrollo 47. Santiago de Chile: Comisión Económica para América Latina y el Caribe, Naciones Unidas.

RODRIGUEZ, A. \& DURAN, F., 2000. Costos e Incentivos en la Organización de un Sistema de Pensiones. Serie Financiamento del Desarrollo 98. Santiago de Chile: Comisión Económica para América Latina y el Caribe, Naciones Unidas.

SOLIMANO, G.; VERGARA, M. \& ISAACS, S., 1999. Las Reformas de Salud en América Latina: Fundamentos y Análisis Crítico. Santiago de Chile: Corporación de Salud y Políticas Sociales.

SUPERSALUD (Superintendencia Nacional de Salud), 1996. Recursos Financieros del Sector Salud. Bogotá: Ministero de Salud.

WAGSTAFF, A. \& van DOORSLAER, E., 1992. Equity in the finance and delivery of health care: Concepts and definitions. In: Equity in the Finance and De livery of Health Care An International Perspective (A. Wagstaff, E. van Doorlaer, \& F. Rutten, ed.), pp. 85-103, Oxford: Oxford University Press.

WAGSTAFF, A.; van DOORSLAER, E. \& BACI, P.,1991. Equity in the finance and delivery of health care: Some tentative cross-Country comparisons. In: Providing Health Care: The Econimics of Alternative Systems of Financing and Delivery (A. McGuire, P. Fenn \& K. Mayhew, ed.), pp. 141-171, Oxford: Oxford University Press.

WHO (World Health Organization), 2000. TheWorld Health Report 2000. Health Systems: Improving Performance. Geneva: WHO.

WOOLHANDLER, S. \& HIM MELSTEIN, D. U., 1991. The deteriorating administrative efficiency of the US health care system. New England Journal of Medicine, 324:1253-1258.

WORLD BANK, 1993. World Development Report 1993. Investing in Health. Oxford: Oxford University Press.

WORLD BANK, 2000. World Development Report 2000/2001. Atacking Poverty. Oxford: Oxford University Press.

Recibido el 1 de junio de 2001

Versión final presentada el 1 de junio de 2001

Aprobado el 10 septiembre de 2001 